\title{
Search for top squark pair production in final states with two leptons at LHC Run 2 with the ATLAS detector
}

\section{Luigi Longo, On behalf of the ATLAS Collaboration}

INFN Sezione di Lecce and Dipartimento di Matematica e Fisica E. De Giorgi dell'Università del Salento, Italy.

E-mail: luigi.longo@cern.ch

\begin{abstract}
Although no experimental evidence has been found during LHC Run1, Supersymmetry (SUSY) remains one of the most promising and motivated Standard Model (SM) extensions. Focusing the attention on models where the multiplicative quantum number R-parity is conserved, the latest results in searching for pair production of top squarks decaying to a bottom quark and the lightest chargino or to a top quark and the lightest supersymmetric particle (neutralino) in final states with 2 leptons are presented, using proton-proton collision data collected by the ATLAS experiment during 2015 and 2016 at a center-of-mass energy of $13 \mathrm{TeV}$ and corresponding to an integrated luminosity of $36.1 \mathrm{fb}^{-1}$. Each of the decay modes is searched in the context of a simplified model, assuming a branching ratio of $100 \%$ for both signals.
\end{abstract}

EPS-HEP 2017, European Physical Society conference on High Energy Physics

5-12 July 2017

Venice, Italy 


\section{Introduction}

Even if the Standard Model (SM) of particle physics describes in a successful way the elementary particles and the electromagnetic, weak and strong interactions, there are still several open questions, such as the nature of the dark matter or the hierarchy problem, suggesting that a more general theory is needed. Among the possible SM extensions, the Minimal Supersymmetric Standard Model (MSSM) is one of the most studied frameworks given that it provides a natural solution to both problems mentioned before, once assuming the conservation of the multiplicative quantum number $R$ - parity $^{1}$. In this paper, the results of the search of the supersymmetric partner of the top quarks, $\tilde{t}_{1}$, in a final state with 2 leptons with the ATLAS detector [1] are presented and discussed.

Two different signal models have been considered for the analysis optimization [2] and both of them assume the lightest neutralino, $\tilde{\chi}_{1}^{0}$, as the lightest supersymmetric particle (LSP). While in the first one the top squark decays into a top quark, $t$, and the lightest neutralino, $\tilde{\chi}_{1}^{0}$, the second one supposes a $\tilde{t}_{1}$ decaying into a bottom quark, $b$, and the lightest chargino, $\tilde{\chi}_{1}^{ \pm}$, which in turn decays into a $W$ boson and a $\tilde{\chi}_{1}^{0}$. For both decay processes a branching ratio of $100 \%$ has been considered.

All the proton-proton collision data, delivered by the Large Hadron Collider (LHC) [3] and collected by the ATLAS detector during 2015 and 2016, at a center of mass energy of $13 \mathrm{TeV}$ and corresponding to an integrated luminosity of $36.1 \mathrm{fb}^{-1}$ have been used for this analysis.

\section{Analysis strategy}

Since the kinematic properties of each signal model are strongly correlated with the splitting in mass, $\Delta m$, between the mother particle $\left(\tilde{t}_{1}\right)$ and its decay products $\left(\tilde{\chi}_{1}^{ \pm}\right.$or $\left.\tilde{\chi}_{1}^{0}\right), 3$ different signal regions (SR) have been developed:

- SRA targets $\tilde{t}_{1}$ decaying into $b \tilde{\chi}_{1}^{ \pm}$with a splitting in mass between top squark and chargino equal to $10 \mathrm{GeV}$; for these models the b-jets from the top squarks are too soft to be reconstructed and no b-tagged jet can be consequently requested.

- SRB focuses on signal models with large $\Delta m$ and for which it is possible to tag the jets coming from the $b$-quarks hadronisation.

- SRC looks at the decays into $t \tilde{\chi}_{1}^{0}$ in scenarios where $m_{\tilde{t}_{1}} \sim m_{t}+m_{\tilde{\chi}_{1}^{0}}$; these signals show a behavior similar to the $t \bar{t}$ processes especially when the top squarks are produced at rest and the momenta carried by the neutralinos in the final state are small. For these cases a better separation can be obtained requiring an initial state radiation (ISR) jet that recoils again the top squarks pair.

All SRs mainly rely on the lepton-based stransverse mass, $\mathrm{m}_{T 2}$, variable ${ }^{2}$ and for each $\mathrm{SR}$ a different optimization has been done for events having a same flavor (SF) or different flavor (DF)

\footnotetext{
${ }^{1}$ The multiplicative quantum number $R$-parity is defined as $(-1)^{3(B-L)+2 S}$ with $B, L$ and $S$ respectively baryon number, leptonic number and spin of the particle. If the $R$-parity is conserved, SUSY particles are produced in pairs and the lightest supersymmetric particle (LSP) is stable and is a good candidate for the dark matter.

${ }^{2}$ The lepton-based $m_{T 2}$ is defined as $\min _{\vec{q}_{t}+\vec{r}_{t}=\vec{P}_{t}^{m i s s}}\left\{\max \left[m_{T}\left(\vec{P}_{t}^{l_{1}}, \vec{q}_{t}\right), m_{T}\left(\vec{P}_{t}^{l_{2}}, \vec{r}_{t}\right)\right]\right\}$ where $\vec{P}_{t}^{l_{1}}, \vec{P}_{t}^{l_{2}}$ are, respectively, the transverse momenta of the leading and subleading leptons, $\vec{P}_{t}^{\text {miss }}$ is the missing transverse momentum, $\vec{q}_{t}$ and $\vec{r}_{t}$ are the transverse momentum vectors so that $\vec{q}_{t}+\vec{r}_{t}=\vec{P}_{t}^{\text {miss }}$ while $m_{T}$ is the transverse mass.
} 
leptons final state. In Fig. 1 the lepton-based stransverse mass distributions for SRA DF and SRC SF before applying the final $\mathrm{m}_{T 2}$ cut are shown.
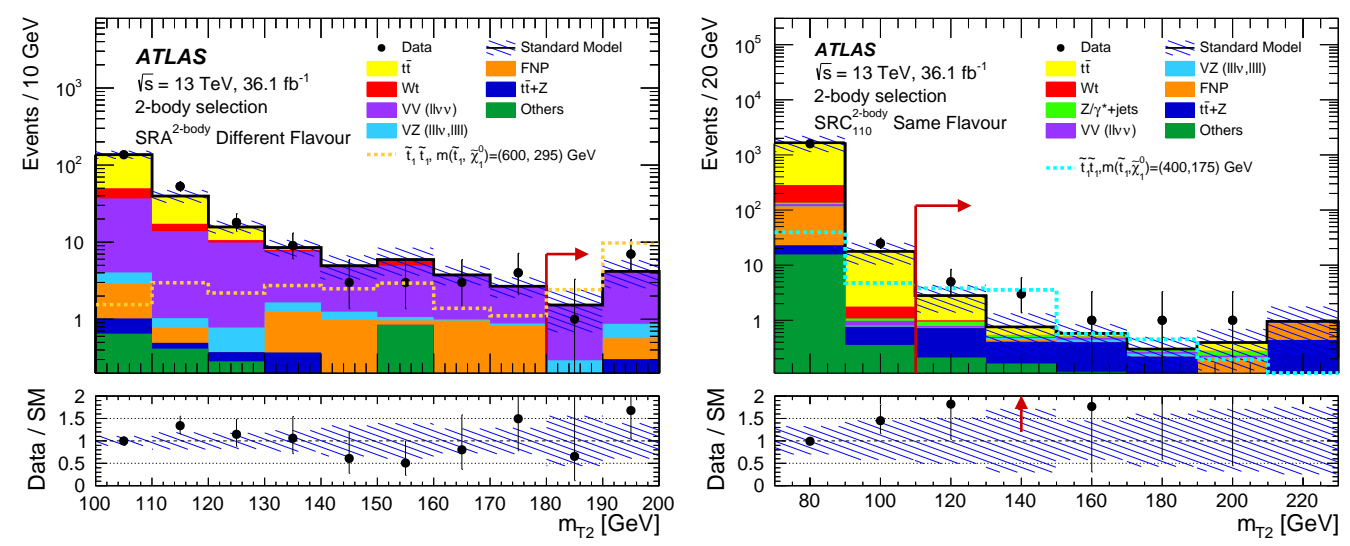

Figure 1: The distribution of $\mathrm{m}_{T 2}$ for events satisfying the selection criteria of SRA DF (left) and SRC $\mathrm{SF}$ (right) with the exception of the $\mathrm{m}_{T 2}$ cut. The contributions of all the SM backgrounds are shown as histogram stack; the error band takes into account the total statistical and systematic uncertainty. The rightmost bin of each plot includes overflow events. Reference signal models are overlayed for comparison. Red arrows indicate the cut applied for the signal region definition [2].

Table 1: The observed and estimated SM events, the model-independent 95\% CL upper-limits on the visible cross-section $\left(\sigma_{\mathrm{vis}}\right)$ of new physics and the discovery $p$-value $(p(s=0))$, all calculated with pseudoexperiments, are shown for each SR [2].

\begin{tabular}{lcccc}
\hline Signal Region & Observed events & Estimated SM events & $\sigma_{\text {vis }}[\mathrm{fb}]$ & $p(s=0)$ \\
\hline SRA SF & 16 & $12.3 \pm 2.3$ & 0.37 & 0.20 \\
SRA DF & 8 & $5.4 \pm 1.7$ & 0.26 & 0.19 \\
SRB SF & 9 & $7.4 \pm 1.1$ & 0.24 & 0.28 \\
SRB DF & 7 & $4.8 \pm 1.0$ & 0.23 & 0.19 \\
SRC SF & 11 & $5.3 \pm 1.8$ & 0.36 & 0.05 \\
SRC DF & 7 & $3.8 \pm 1.5$ & 0.26 & 0.12 \\
\hline
\end{tabular}

\section{Interpretation}

No significant excess has been observed in any SRs and model-independent upper-limits have been estimated on the visible cross-section as reported in Tab.1. Limits on the simplified models $\tilde{t}_{1} \rightarrow t \tilde{\chi}_{1}^{0}$ and $\tilde{t}_{1} \rightarrow b \tilde{\chi}_{1}^{ \pm}$, with $\Delta m\left(\tilde{t}_{1}, \tilde{\chi}_{1}^{ \pm}\right)$equal to $10 \mathrm{GeV}$, have been also computed together with limits on a pMSSM model where the wino and bino mass parameters, respectively $M_{1}$ and $M_{2}$, are set to $M_{2}=2 M_{1}$ with $m_{\tilde{t}_{1}}>m_{\tilde{\chi}_{1}^{ \pm}}$(Fig. 2). 

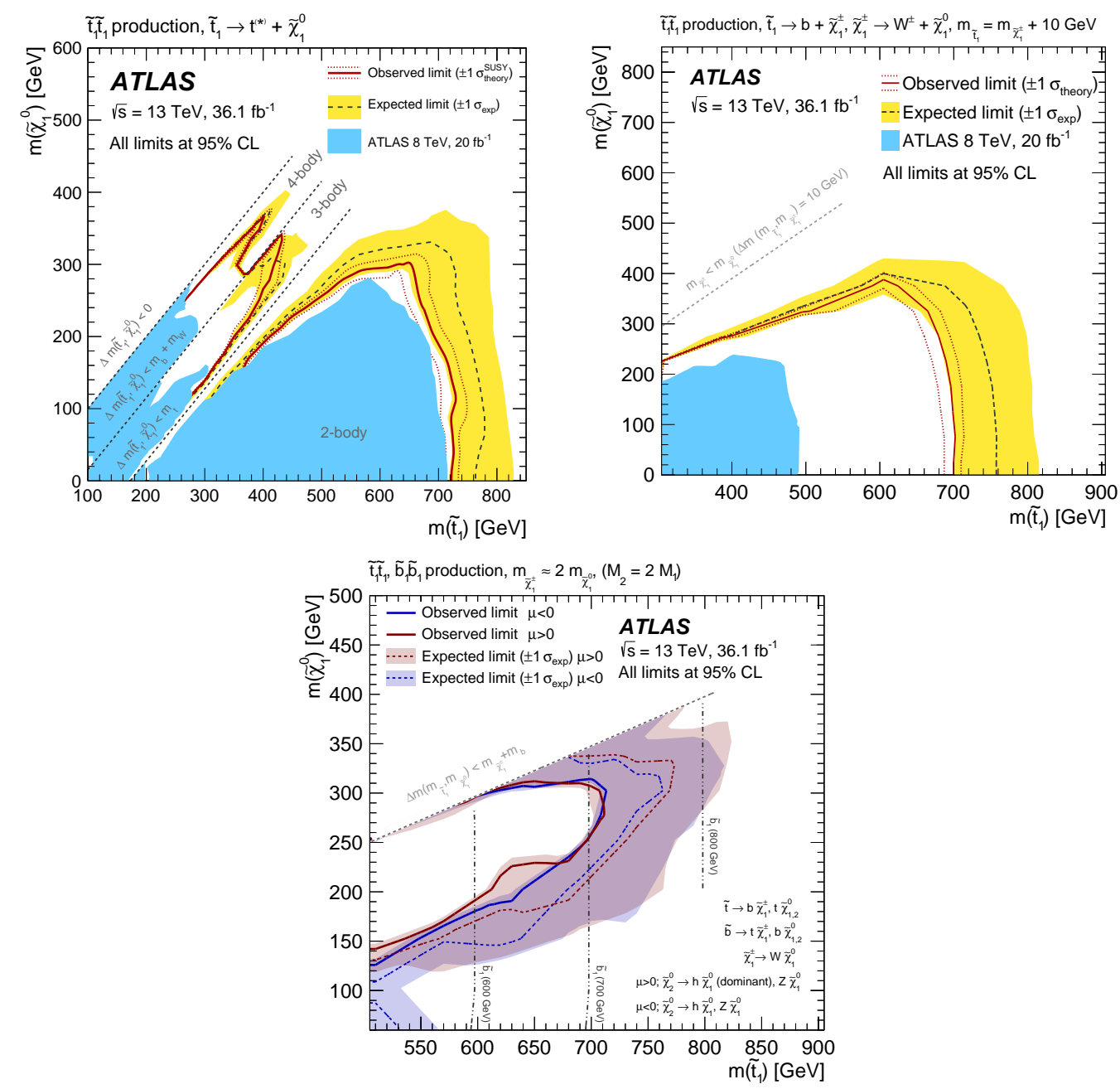

Figure 2: Exclusion contours for the simplified models $\tilde{t}_{1} \rightarrow t \tilde{\chi}_{1}^{0}$ (top-left) and $\tilde{t}_{1} \rightarrow b \tilde{\chi}_{1}^{ \pm}$(top-right) and for the pMSSM model (bottom) described in the text. For each plot the dashed line and the shaded band are the expected limit and its $\pm 1 \sigma$ uncertainty; the thick solid line is instead the observed limit for the central value of the signal cross-section. For the pMSSM model pair production of top and bottom squark is considered and limits are set for both the positive (red line in figure) and negative (blue line in figure) values of $\mu$ [2].

\section{References}

[1] ATLAS Collaboration, 2008 JINST 3 S08003.

[2] ATLAS Collaboration, Search for direct top squark pair production in final states with two leptons in $\sqrt{s}=13 \mathrm{TeV}$ pp collisions with the ATLAS detector, arXiv:1708.03247 [hep-ex].

[3] L. Evans and P. Bryant (editors) 2008, JINST 3 S08001. 